\section{Astrocytes, microglie et plasticité synaptique}

\section{Rémi Ronzano}

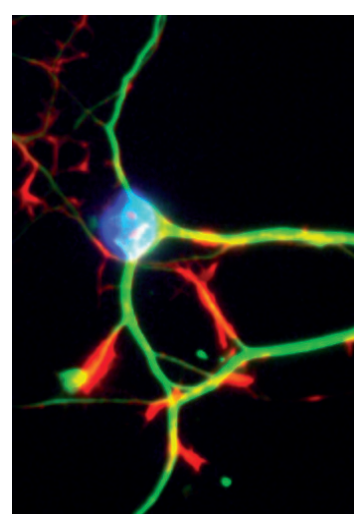

École normale supérieure de Cachan, université ParisSaclay, 61, avenue du Président Wilson, 94235 Cachan Cedex, France.

remi.ronzano@ens-paris-saclay.fr neuronal, via la recapture des neurotransmetteurs et la libération de nombreux facteurs dont certains modifient l'état d'inflammation. Par ailleurs, l'environnement cellulaire peut lui-même entraîner des modifications de l'expression de ces facteurs gliaux. Ainsi, dans le système nerveux central, ces populations de cellules «non neuronales» sont impliquées dans les mécanismes de plasticité synaptique. Cette revue s'attachera à décrire les modulations des mécanismes de potentialisation et de dépression à long terme induites par la glie ainsi que le rôle de la signalisation entre astrocytes et microglie sur ces mécanismes. <

Les synapses sont des structures plastiques qui s'adaptent aux changements d'activité des réseaux neuronaux en modifiant leur composition et leur organisation. Deux de ces modifications ont largement été étudiés: la PLT (ou potentialisation à long terme) et la DLT (ou dépression à long terme). Ces deux mécanismes, sur lesquels cette revue se concentrera, dépendent de l'activité du réseau neuronal et modifient de façon durable la force synaptique, c'est-à-dire l'effet d'un potentiel d'action sur l'amplitude du courant postsynaptique. PLT et DLT sont des mécanismes qui peuvent impliquer les

Vignette (Photo @ Inserm-Yasmina Saoudi et Sandrine Ballet). compartiments pré- ou postsynaptique : au niveau présynaptique, par un changement dans la libération des neurotransmetteurs; au niveau postsynaptique, par une modification du nombre des récepteurs canaux présents, de leurs propriétés et de leur localisation. La plasticité à long terme peut également reposer sur des modifications structurales de la synapse, via une réorganisation du cytosquelette. La PLT et la DLT ont été décrites au niveau des synapses excitatrices et des synapses inhibitrices. Des mécanismes comparables sont également connus pour les synapses électriques. De nombreux travaux ont montré l'importance de ces mécanismes dans l'encodage de la mémoire, au niveau de différentes structures du système nerveux central. Leur rôle dans les mémoires déclarative et non déclarative, c'est à dire dans l'acquisition consciente des souvenirs et des capacités motrices et cognitives, est maintenant largement admis. La description fine de ces mécanismes et de leur modulation, comme l'identification des acteurs impliqués, apparaît ainsi essentielle à la compréhension des processus neuronaux présidant à la formation de la mémoire.

La mise en évidence de signaux échangés entre astrocytes et neurones, associée à l'accumulation des données montrant la proximité et le rôle des astrocytes des synapses, ont conduit au concept de synapse tripartite, en référence au modèle précédemment considéré de synapse bipartite, intégrant exclusivement les structures neuronales (Figure 1) [1] $(\rightarrow)$.

$(\rightarrow)$ Voir la Nouvelle de A. Panatier et R. Robitaille, $m / s$ $n^{\circ}$ 6-7, juin-juillet 2012, page 582 Anatomiquement, seule une fraction des synapses du système nerveux central présente, cependant, une telle organisation tripartite.

L'étude récente des cellules microgliales, cellules immunitaires d'origine macrophagique présentes au sein du système nerveux central, a révélé leur importance et leur proximité avec les synapses (Figure 1), 


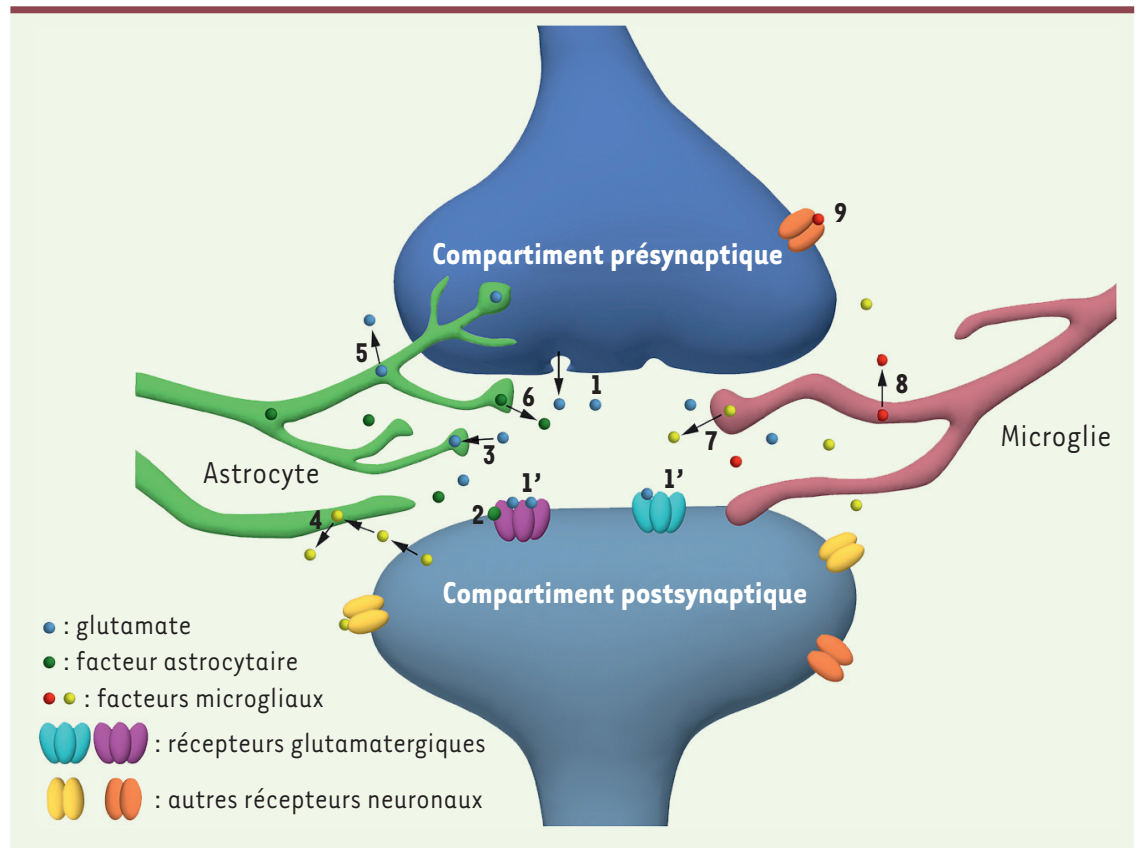

Figure 1. Organisation de la synapse et cellules gliales. Un compartiment présynaptique libérant un neurotransmetteur, par exemple le glutamate (1), active différents récepteurs au niveau postsynaptique (1') avec l'implication éventuelle de facteurs d'origine astrocytaire (2). Autour de la synapse, peuvent être présents des prolongements cytoplasmiques astrocytaires et/ ou microgliaux. Les astrocytes recapturent le glutamate et d'autres composés présents dans l'environnement synaptique (3, 4), libèrent des neurotransmetteurs (5) et d'autres facteurs (6). La microglie produit également des facteurs qui sont différents selon qu'elle soit dans un état inactivé (7) ou activé (8). Les facteurs libérés peuvent aussi agir sur d'autres récepteurs pré- et postsynaptiques (9).

ce qui a soulevé la question de leur rôle potentiel dans les modulations synaptiques [2]. La microglie, longtemps considérée pour ne présenter une activité qu'en conditions pathologiques (état activé) est, en réalité, également impliquée dans des mécanismes physiologiques, développementaux, et à l'âge adulte (état inactivé). Elle participe, notamment, à la formation et à l'élimination des synapses, au contrôle des processus apoptotiques, et à la libération de nombreux facteurs qui agissent sur les réseaux neuronaux.

La microglie dérive des macrophages primitifs qui sont présents dans le sac vitellin. Au cours du développement, elle envahit le système nerveux central via le système sanguin. Les cellules astrocytaires, quant à elles, se différencient à partir du neuroépithélium.

Les modulations exercées par ces cellules se sont révélées beaucoup plus diversifiées qu'envisagé auparavant. Leurs implications dans les mécanismes de plasticité ne sont aujourd'hui plus remises en cause, et de récents travaux ont apporté des connaissances nouvelles sur leur rôle dans les modulations synaptiques directes et indirectes. Leur interaction avec la synapse, et l'environnement synaptique qu'elles partagent, font de ces cellules des actrices essentielles des mécanismes synaptiques. Leurs interactions au niveau synaptique pourraient également être impliquées, de façon indirecte, dans la modulation des mécanismes de plasticité à long terme.

\section{Les astrocytes : des acteurs de la plasticité synaptique}

Des expériences d'implantation corticale d'astrocytes immatures avaient montré que ces cellules induisaient une augmentation de la plasticité de cette structure [3]. Depuis, de nombreux travaux ont confirmé l'existence d'une modulation astrocytaire de la plasticité synaptique, notamment par la libération de différents facteurs.

\section{Facteurs astrocytaires influençant la plasticité}

\section{La D-sérine}

La D-sérine se lie au niveau des sites de liaison de la glycine et module l'activité des récepteurs NMDA (récepteur du N-méthyl-D-aspartate). Elle est synthétisée à partir de la L-sérine par une racémisation catalysée par la sérine racémase, qui est exprimée au niveau astrocytaire et neuronal [4]. En modulant l'activité des récepteurs NMDA, la D-sérine d'origine astrocytaire agit sur les mécanismes de PLT [5], probablement par l'intermédiaire de signaux calciques [6], bien que cela soit sujet à controverses [7]. L'intervention de ces signaux est suggérée par la diminution de la PLT que l'on observe au niveau de l'aire CAl (cornu ammonis 1 area, ou corne d'Ammon) de l'hippocampe à la suite de la délétion, ou du blocage, du canal TRPAl (transient potential receptor Al) impliqué dans la régulation du taux de calcium $\left(\mathrm{Ca}^{2+}\right)$ dans les astrocytes [8] (Figure 2). L'étude d'autres structures a également permis la démonstration du rôle de la D-sérine d'origine astrocytaire dans la PLT : dans le noyau basal de Meynert, par exemple, sa libération par les astrocytes, induite par l'acétylcholine, déclenche un signal calcique [9]; sa libération peut également avoir un effet sur les mécanismes de DLT au niveau du noyau supraoptique [10] et au niveau du cervelet, par son action sur le récepteur ionotropique au glutamate $\delta 2$ [11] (Figure 2).

Le rôle de la $D$-sérine dans la plasticité synaptique et l'intervention astrocytaire dans sa régulation ne sont plus remis en question. Cependant, le modèle de 


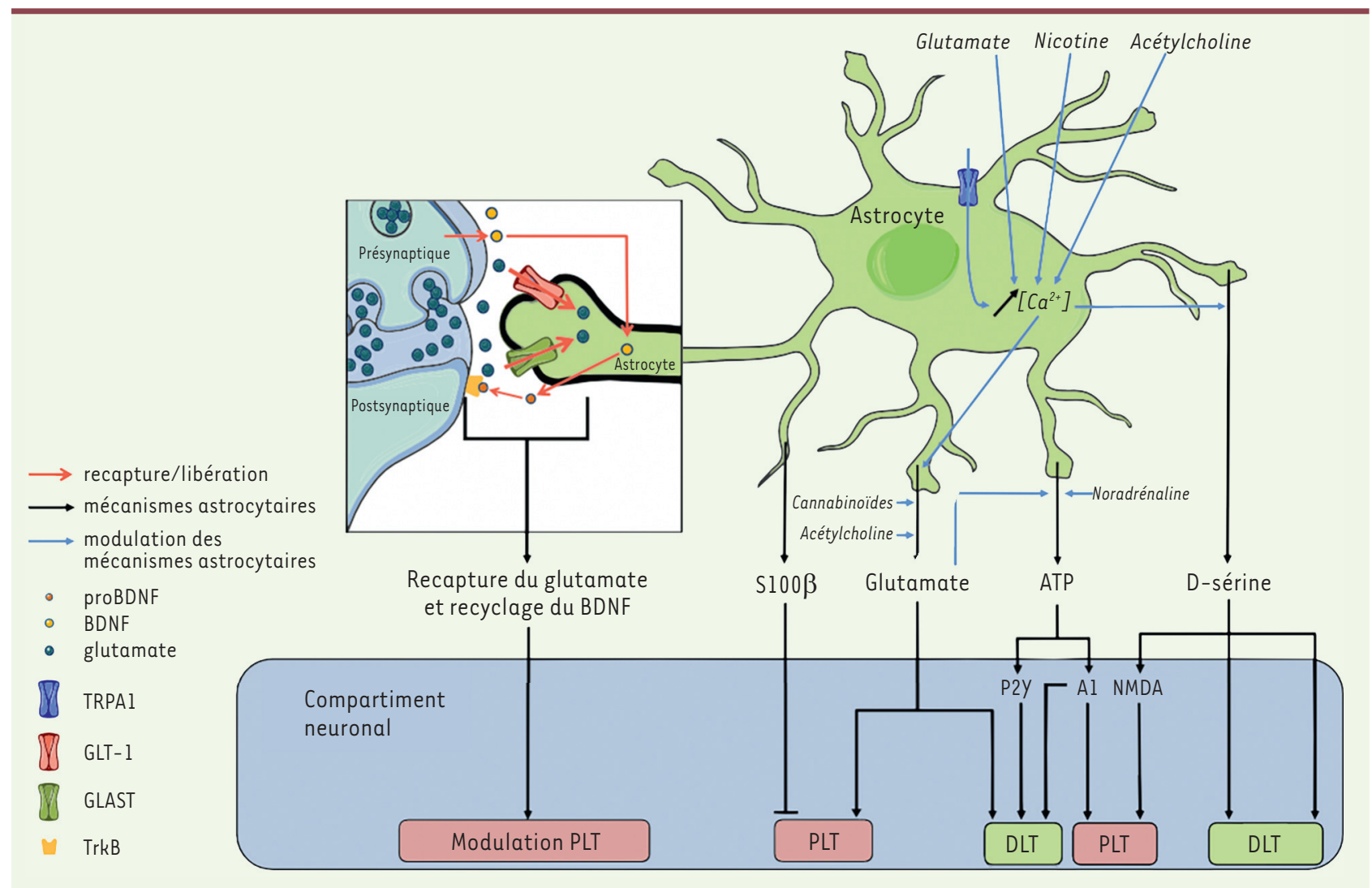

Figure 2. Rôle des astrocytes dans la plasticité synaptique. Les astrocytes via la libération de facteurs, la réabsorption du glutamate et le recyclage du BDNF (brain derived neurotrophic factor) à la synapse modulent les mécanismes de potentialisation à long terme (PLT) et de dépression à long terme (DLT). Ces mécanismes de libération peuvent être déclenchés par différentes voies de signalisation. TrkB : tropomyosin-related kinase B ; ATP : adenosine tri-phosphate ; NMDA : N-methyl-D-aspartate; P2Y : récepteur P2Y ; $\mathrm{Al}$ : récepteur Al de l'adénosine ; TRPAl : transient potential receptor Al ; GLT-1 : glutamate transporter 1 ; GLAST : glutamate aspartate transporter.

libération de la D-sérine et son origine neuronale ou astrocytaire restent très débattus.

\section{Le glutamate}

Le glutamate est, au niveau des synapses excitatrices, le neurotransmetteur majeur du système nerveux central. Très majoritairement libéré par les neurones glutamatergique au niveau de la synapse, il peut être libéré par les astrocytes, notamment en réponse à une signalisation calcique [12]. Sa libération par ces cellules, est impliquée dans l'activation de la PLT et de la DLT au niveau de différentes structures [1315]. Au niveau cortical, la sécrétion de glutamate astrocytaire, qui déclenche la DLT, repose sur une signalisation endocannabinoïde [14]. Au niveau de la région CAl, la libération de glutamate astrocytaire, qui est impliquée dans la PLT, est induite par une signalisation cholinergique [15]. Les voies de signalisation pouvant déclencher la libération de glutamate semblent donc multiples (Figure 2).

\section{L'adénosine}

Le glutamate [16], ou la noradrénaline, déclenchent la production d'ATP (adénosine triphosphate) par les astrocytes. Néanmoins, les mécanismes impliqués dans cette libération restent sujets à polémique. Cette libération pourrait se produire de manière dépendante de signaux calciques par exocytose [17], ou indépendamment par activation du récepteur ionotropique perméable aux cations, $\mathrm{P} 2 \mathrm{X}_{7}$ [18] et/ou d'hémicanaux qui permettent la diffusion de métabolites comme I'ATP, du cytoplasme vers le milieu extracellulaire. L'ATP et l'adénosine, provenant de sa dégradation par des ectonucléotidases, participent à la communication entre les cellules gliales et les neurones [19]. Ils modulent la plasticité par de nombreux mécanismes comme le déclenchement de synaptic scaling (plasticité homéostatique, modulant la force synaptique au niveau de multiples synapses) [16], l'augmentation de la force synaptique [18], et la plasticité synaptique à court terme. L'adénosine, dérivant de l'ATP libéré, contrôle également l'amplitude disponible pour les mécanismes de PLT. Elle module la DLT hétérosynaptique par l'intermédiaire du récepteur Al qui participe par ailleurs, à la modulation de la 
libération des neurotransmetteurs [17]. Les récepteurs métabotropiques P2Y seraient, eux aussi, impliqués dans la DLT hétérosynaptique [20], un mécanisme permettant une potentialisation ciblée (Figure 2).

\section{Conclusion intermédiaire}

La signalisation conduisant à la libération de ces facteurs, de même que l'importance des astrocytes dans ces mécanismes, restent largement incomprises [4, 21, 22]. Les signaux calciques, souvent mis en cause dans ces processus de libération, sont en fait perturbés lorsqu'ils sont examinés à l'échelle cellulaire, et leur implication à l'échelle subcellulaire reste, pour le moment, à clarifier. La description précise de ces signaux et de leur localisation au cours des mécanismes de libération demeure donc un enjeu majeur pour la compréhension fine de la fonction des astrocytes. Plus généralement, le rôle de ces signaux dans la plasticité et l'ensemble des mécanismes sous influence gliale au niveau central reste à déterminer.

\section{Recapture des neurotransmetteurs et recyclage à la synapse Le glutamate}

La recapture du glutamate au niveau synaptique est réalisée par les neurones et majoritairement par les astrocytes qui expriment des transporteurs, le principal étant GLT-l (glutamate transporter 1). De par leur contrôle sur la concentration et la diffusion du glutamate au niveau de la fente synaptique, les astrocytes sont directement impliqués dans la modulation de l'activité synaptique et des mécanismes de plasticité. Au niveau du striatum, la recapture du glutamate de la fente synaptique via GLT-1 permet de contrôler la temporalité de la plasticité synaptique [23]. Dans I’hippocampe, la surexpression de GLT-l affecte les mécanismes de DLT des synapses entre les fibres moussues, issues du gyrus denté, et les cellules pyramidales de l'aire CA3 [24]. La délétion du gène codant le récepteur EphA4 (A4 type ephrin receptor) ou d'un de ces ligands, l'EphA3 (ephrinA3) normalement impliqués dans la modulation de la structure dendritique et de la plasticité, entraîne, chez la souris, une augmentation de l'expression par les astrocytes des transporteurs de glutamate GLT-l et GLAST (glutamate aspartate transporter) à l'origine de l'inhibition de la PLT au niveau de l'aire CAl de l'hippocampe [25]. Ces résultats renforcent alors l'hypothèse d'une fonction importante de ces transporteurs dans la plasticité synaptique (Figure 2).

\section{Le BDNF}

Les astrocytes participent à la recapture du BDNF (brain-derived neurotrophic factor), un facteur neurotrophique abondant au niveau du cerveau. Le BDNF est notamment impliqué dans la survie, la différenciation et la plasticité neuronale [26]. Il est produit par les neurones sous la forme d'un précurseur (le proBDNF) qui favorise la DLT en agissant sur le récepteur pan-neurotrophique p75NTR. Sous sa forme mature (BDNF), il facilite la PLT en agissant sur le récepteur postsynaptique TrkB (tropomyosin-related kinase $B$ ) tout en prévenant l'induction de la DLT. Le recyclage du proBDNF par les astrocytes au niveau de la synapse par sa recapture, sa maturation sous forme de BDNF et sa libération, est essentiel au maintien de la phase tardive de la PLT
[27]. Les astrocytes apparaissent donc être des acteurs indispensables de cette signalisation (Figure 2).

\section{Les cellules microgliales, actrices de la plasticité synaptique}

À l'âge adulte, la déplétion des cellules microgliales entraîne, chez la souris, une modification significative de l'expression de nombreuses protéines, dont vingt et une sont impliquées dans la plasticité synaptique [28], ce qui renforce l'hypothèse de la participation de la microglie à la modulation de la plasticité.

\section{En situation non pathologique, modulation par le BDNF}

L'inactivation du gène codant le BDNF spécifiquement dans les cellules microgliales, conduit à une inhibition de la plasticité structurale [28]. La protéine adaptatrice KARAP (killer cell activating receptor-associated protein ou DAP12 pour DNAX activating protein of 12 $k D a)$ exprimée par des cellules lymphoïdes et myéloïdes module l'activation microgliale. Les souris Karap/ Dap12 $2^{-/}$, qui ne produisent pas KARAP, présentent une diminution de l'expression des récepteurs TrkB et une augmentation de la PLT [29]. Le lien direct entre BDNF d'origine microgliale et modulation de la PLT reste cependant à vérifier.

\section{En situation pathologique, libération de facteurs} influençant la plasticité synaptique

Au cours du processus inflammatoire neuronal, la microglie adopte un état dit activé; les cellules changent leur spectre d'expression, libérant des facteurs différents de ceux qu'elles produisent en situation physiologique qui sont également impliqués dans la modulation de la plasticité synaptique.

\section{Le TNF $\alpha$, à forte concentration}

Le TNF $\alpha$ (tumor necrosis factor $\alpha$ ) est une cytokine au rôle ambivalent au niveau du système nerveux central. Elle peut être pro- ou anti-inflammatoire selon les conditions. Dans le système nerveux central, elle est libérée constitutivement par la microglie en petite quantité, mais en quantité beaucoup plus importante dans les situations inflammatoires. Le TNF $\alpha$, exprimé comme une protéine transmembranaire qui peut être convertie sous une forme soluble par la métalloprotéase TACE (TNF $\alpha$-converting enzyme), est actif sous ces deux formes. II interagit avec deux récepteurs, TNFRl (récepteur du TNFl), souvent associé à des signalisations pro-inflammatoires, et TNFR2 (récepteur du TNF2), dont la signalisation est souvent liée à la réparation 


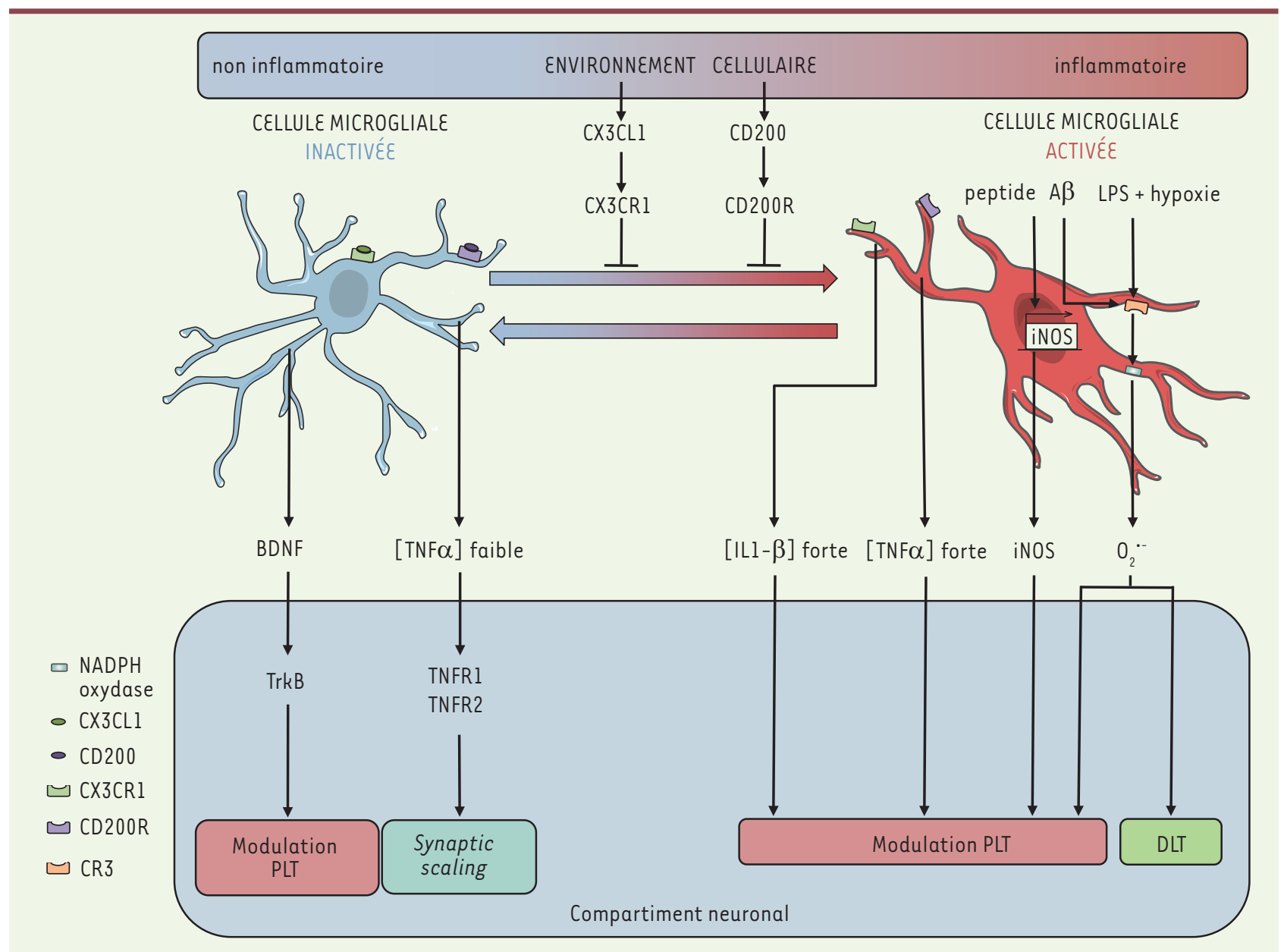

Figure 3. Modulation des mécanismes de plasticité synaptique par les cellules microgliales. La microglie libère différents facteurs impliqués dans la modulation des mécanismes de plasticité synaptique. Le spectre des facteurs délivrés et ayant la propriété de moduler les mécanismes de plasticité à long terme est dépendant de l'état d'activation de la microglie ; la modulation de l'état d'activation est donc indirectement impliquée dans ces mécanismes. BDNF : brain derived neurotrophic factor; PLT : potentialisation à long terme; DLT : dépression à long terme; CX3CLI : chemokine [C-X3-C motif] ligand 1 ; CX3CRI : CX3C chemokine receptor 1 ; CR3 : récepteur du complément 3 ; TrkB : tropomyosin-related kinase B ; iNOS : N0 synthase inductible; LPS : lipopolysaccharide.

neuronale. La synthèse de TNF $\alpha$ par les astrocytes, évoquée initialement, est désormais controversée par des données transcriptomiques et par les possibles contaminations des cultures astrocytaires par des cellules microgliales qui libèrent, elles aussi, la cytokine [30]. L'origine gliale du TNF $\alpha$ n'est donc pas complètement admise [31]. Les souris invalidées pour le gène codant le TNF $\left(\mathrm{Tnf}^{-{ }^{-}}\right)$ou celles qui n'expriment pas son récepteur, présentent une diminution du mécanisme de synaptic scaling au niveau de l'hippocampe et du cortex visuel. Elles ne montrent cependant pas de modification des mécanismes de PLT ou de DLT [32] (Figure 3). Le traitement de tranches d'hippocampes par le TNF $\alpha$, à des concentrations physiopathologiques, semble néanmoins moduler ces mécanismes. Ainsi, à faibles concentrations, le TNF $\alpha$ ne paraît pas avoir d'effet sur la PLT et la DLT, ce qui explique les résultats observés chez les souris $\mathrm{Tnf}^{-{ }^{--}}$, ou celles qui n'expriment pas de récepteur pour le TNF $\alpha$. Cependant, en conditions inflammatoires (sous un traitement avec de fortes concentrations de TNF $\alpha$ ), le mécanisme de PLT serait modifié (Figure 3). L'effet de ce facteur sur la plasticité synaptique reste donc à clarifier, et la description des voies de signalisation activées, respectivement à faible et à forte concentration de TNF $\alpha$, apparaît essentielle.

\section{La NADPH oxydase}

La NADPH (nicotinamide adenine dinucleotide phosphate) oxydase est une enzyme qui active par différentes voies, en situation d'hypoxie ou d'inflammation, la production d'anions superoxydes. L'activation de cette enzyme en situation inflammatoire, induit l'inhibition de la PLT, celle-ci pouvant être levée lorsque l'enzyme est inhibée [33]. Un traitement avec du 
lipopolysaccharide (LPS) bactérien, qui stimule les voies pro-inflammatoires des cellules, associé à une hypoxie, permet d'induire un mécanisme de DLT qui dépend de l'activation de la NADPH oxydase [34]. La NADPH oxydase microgliale participerait donc à la modulation des mécanismes de PLT et de DLT en situation inflammatoire (Figure 3).

\section{La iNOS}

La iNOS (inducible nitric oxide synthase, ou nitric oxide synthase 2) catalyse la production d'oxyde nitrique à partir de L-arginine. Elle est impliquée dans la lutte anti-microbienne et anti-virale et dans le contrôle de la différenciation des lignées myéloïde et lymphoïde. Cette enzyme est exprimée par la microglie et par les neurones, mais son expression par les astrocytes reste controversée [35]. Au niveau du gyrus denté, un traitement par le peptide $A \beta$ amyloïde conduit à une inhibition de la PLT, ce qui n'est pas observé chez les souris invalidées pour Nos2, le gène codant la iNOS [33]. L'expression de Nos2 par les cellules microgliales semble donc participer à la modulation de la PLT (Figure 3). Néanmoins, il est difficile de déterminer si l'effet que l'on observe a pour origine son expression par les cellules microgliales, ou par les neurones, ou par les deux populations.

\section{Contrôle de l'activation microgliale et modulation de la plasticité synaptique}

En situation inflammatoire, la transition de l'état dit inactivé à l'état activé des cellules microgliales altère l'expression de différents gènes, dont certains sont impliqués dans la modulation de la plasticité synaptique. Le contrôle de l'activation microgliale peut donc impacter indirectement les mécanismes de plasticité synaptique.

\section{Le récepteur $\mathrm{CX} 3 \mathrm{CR} 1$}

CX3CRI (C-X3-C motif receptor 1 ) est un récepteur de chimiokine qui est exprimé par les cellules microgliales. La fixation de son ligand, le CX3CL1 ( $C-X 3-C$ motif ligand 1 ), participe au maintien des cellules microgliales dans un état inactivé [36] (Figure 3). Les souris invalidées pour ce récepteur $\left(\mathrm{C}_{3} 3 \mathrm{crl}^{-/-}\right)$, âgées de 13 jours, présentent une augmentation de la DLT au niveau de l'hippocampe [37]. À l'âge adulte, elles montrent une modification de la PLT et une concentration plus élevée d'IL-1 $\beta$ au niveau du système nerveux central. L'injection d'IL-lra, un antagoniste des récepteurs de l'IL-1 $\beta$, abolit les modifications de la PLT qui sont observées, ce qui renforce l'hypothèse de la participation de cette cytokine (Figure 3).

\section{Signalisation CD200/CD200R}

CD200 est une glycoprotéine membranaire qui participe à l'inhibition de l'activation des cellules microgliales. Au niveau du système nerveux central, elle est exprimée par les neurones et les oligodendrocytes. Elle maintient la microglie dans un état inactivé par son interaction avec son récepteur, le CD200R exprimé par les cellules microgliales [38]. Les souris invalidées pour le gène codant $\operatorname{CD} 200\left(C D 200^{-/-}\right)$présentent ainsi une proportion de cellules microgliales activées plus élevée que des souris sauvages et montrent également une diminution de la PLT, au niveau hippocampique [39] (Figure 3).
L'utilisation de souris CD200 conditional knockout (c'est-à-dire des souris dont la délétion du gène CD200 est inductible) devrait permettre de connaître plus précisément les mécanismes à l'origine de la modification de la plasticité, et d'exclure des modifications durables dues à une activation microgliale forte durant le développement. Ces modifications pourraient indirectement impacter la plasticité synaptique chez l'adulte. Cette hypothèse est en effet envisageable au vu du rôle essentiel de ces cellules et de leur état d'activation dans les processus développementaux.

\section{Signalisation entre astrocytes et cellules microgliales, et plasticité synaptique}

La signalisation induite par la libération par les astrocytes et la microglie de certains facteurs, n'agit pas seulement de la glie vers les neurones. Elle opère également sur les cellules gliales, présentes dans l'environnement synaptique, et influence leurs activités entre elles.

\section{Des astrocytes vers la microglie \\ Rôle de la signalisation S100 $\beta$ sur les cellules microgliales}

La $S 100 \beta$ est une protéine de la famille des protéines de liaison du calcium intracytosolique qui est exprimée spécifiquement au niveau astrocytaire et peut être secrétée. Elle est impliquée dans l'homéostasie calcique, la croissance cellulaire et le métabolisme énergétique. Les souris dont le gène codant la $S 100 \beta$ est délété présentent une augmentation de la PLT au niveau de l'hippocampe [40] (Figure 2). Le mécanisme d'action de la $5100 \beta$ sur la plasticité synaptique n'est cependant pas connu, bien que plusieurs hypothèses puissent être émises. La modulation de la plasticité par la $5100 \beta$ pourrait reposer sur son rôle dans le déclenchement de la signalisation calcique au niveau neuronal et astrocytaire. L'activation du récepteur RAGE (receptor for advanced glycation endproducts) par la $S 100 \beta$ pourrait également initier la libération de cytokines comme le TNF $\alpha$, I'IL(interleukine)-1 $\beta$, I'IL-6, ou l'expression de l'iNOS par les cellules microgliales. Ces partenaires importants de la régulation de la neuroinflammation et la neurodégénération participent également à la modulation de la plasticité synaptique.

\section{Signal ATP astrocytaire agissant sur la microglie}

La libération de BDNF par la microglie peut être déclenchée par I'ATP [28]. II est donc possible que I'ATP secrété par les astrocytes puisse entraîner la libération de BDNF microglial et neuronal, modulant ainsi les 
mécanismes de plasticité synaptique. L'ATP semble également induire, au niveau des fibres $C$ de la moelle épinière, une activation des celIules microgliales par l'intermédiaire du récepteur ionotropique $\mathrm{P} 2 \mathrm{X}_{4}$, et l'induction de mécanismes de PLT [41], ce qui renforce l'hypothèse d'une modulation possible des mécanismes microgliaux par l'ATP d'origine astrocytaire.

\section{Des cellules microgliales vers les astrocytes L'ATP microglial sur les astrocytes}

Les cellules microgliales produisent peu d'ATP. II peut cependant activer les récepteurs adrénergiques astrocytaires $P 2 Y_{1}$ et déclencher une libération d'ATP plus importante par ces dernières [42]. À noter que $P 2 y_{1}$ est impliqué dans l'exocytose du glutamate par les astrocytes [43]. Le récepteur astrocytaire de l'adénosine Al pourrait être également impliqué dans une signalisation d'origine microgliale qui permettrait la régulation de l'expression de GLT-1 [44] qui, lui même, participe à la modulation de la plasticité [23-25]. La libération microgliale d'ATP, quoique faible, pourrait intervenir dans la modulation astrocytaire de la plasticité par l'induction d'une libération d'ATP par ces dernières, et/ou par le contrôle de l'exocytose et la recapture du glutamate.

\section{Le BDNF, signalisation microgliale et recyclage astrocytaire}

Les cellules microgliales ont un rôle dans la plasticité structurale, probablement en l'influençant par la production de BDNF. Le proBDNF libéré par la microglie pourrait, comme le proBDNF d'origine neuronale, être capté, recyclé et transporté au niveau postsynaptique par les astrocytes, modulant ainsi les mécanismes de plasticité [27].

Bien qu'aucune preuve directe du rôle de la communication entre cellules microgliales et astrocytes dans les processus de PLT et DLT n'ait été apportée, certains facteurs d'origine gliale, impliqués dans ces mécanismes, sont libérés en réponse à une signalisation entre astrocytes et microglie. La signalisation intergliale et son implication dans les mécanismes de plasticité reste donc à explorer.

\section{Conclusion}

Aujourd'hui, il est établi que la plasticité synaptique n'est pas un mécanisme uniquement modulé par l'activité des réseaux neuronaux. En revanche, les connaissances de la signalisation aboutissant à la modulation de la plasticité par les cellules gliales et du dialogue intercellulaire intervenant dans ces mécanismes restent partielles. De nombreux facteurs libérés par la microglie participant à la plasticité synaptique ont été mis en évidence, sans qu'un lien direct entre leur libération et la plasticité ne puisse être, pour le moment, établi. Astrocytes et microglie régulent la perméabilité de la barrière hémato-encéphalique. Ces cellules interviennent donc dans les processus modulant l'apport de facteurs du sang vers les neurones, s'inscrivant ainsi comme des acteurs majeurs de la communication entre mécanismes centraux et état physiologique général. Une vision intégrée de la fonction de l'ensemble des acteurs, neurones et cellules gliales, dans la modulation de la plasticité synaptique semble donc nécessaire.
La plasticité est impliquée, en particulier la PLT et la DLT, dans la mémoire et l'apprentissage. La démonstration d'une modulation par la glie de la mémoire et des capacités d'apprentissage, via ses effets sur les mécanismes de PLT et de DLT, nécessite toutefois d'être développée. En revanche, plusieurs études montrent un lien entre modulation de l'apprentissage ou de la mémoire et cellules gliales, et entre cellules gliales et mécanismes de PLT et/ou de DLT [13, 27, 28, 34, 40]. Il semble donc que ces cellules soient impliquées dans les mécanismes d'apprentissage et d'encodage de la mémoire, via leur rôle dans la plasticité, sans exclure une modulation potentielle reposant sur d'autres mécanismes intervenant sur les réseaux neuronaux. $\diamond$

\section{SUMMARY}

Astrocytes and microglia: active players

in synaptic plasticity

Synaptic plasticity consists in a change in structure and composition of presynaptic and postsynaptic compartments. For a long time, synaptic plasticity had been thought as a neuronal mechanism only under the control of neural network activity. However, recently, with the growing knowledge about glial physiology, plasticity has been reviewed as a mechanism influenced by the synaptic environment. Thus, it appears that astrocytes and microglia modulate these mechanisms modifying neural environment by clearance of neurotransmitters, releasing essential factors and modulating inflammation. Moreover, glia can change its own activity and the expression pattern of many factors that modulate synaptic plasticity according to the environment. Hence, these populations of "non-neuronal" cells in the central nervous system seem to be active players in synaptic plasticity. This review discusses how glia modulates synaptic plasticity focusing on long-term potentiation and depression, and questions the role of the signaling processes between astrocytes and microglia in these mechanisms. $\diamond$

\section{LIENS D'INTÉRÊT}

L'auteur déclare n'avoir aucun lien d'intérêt concernant les données publiées dans cet article.

\section{RÉFÉRENCES}

1. Panatier A, Robitaille R. L'astrocyte, un partenaire clé des neurones au cours de la transmission synaptique de base. Med Sci (Paris) 2012 ; 28 : $582-4$

2. Tremblay ME, Majewska AK. A role for microglia in synaptic plasticity? Commun Integr Biol $2011 ; 4: 220-2$.

3. Muller CM, Best J. Ocular dominance plasticity in adult cat visual cortex after transplantation of cultured astrocytes. Nature 1989 ; 342 : 427-30.

4. Kartvelishvily $\varepsilon$, Shleper M, Balan L, et al. Neuron-derived D-serine release provides a novel means to activate $\mathrm{N}$-methyl-D-aspartate receptors. J Biol Chem 2006 ; 281 : 14151-62. 


\section{RÉFÉRENCES}

5. Yang $\mathrm{Y}, \mathrm{Ge} W$, Chen $\mathrm{Y}$, et al. Contribution of astrocytes to hippocampal long-term potentiation through release of D-serine. Proc Natl Acad Sci USA 2003; 100 : 15194-9.

6. Henneberger C, Papouin T, Oliet SH, Rusakov DA. Long-term potentiation depends on release of D-serine from astrocytes. Nature $2010 ; 463: 232-6$.

7. Agulhon C, Fiacco TA, McCarthy KD. Hippocampal short- and long-term plasticity are not modulated by astrocyte Ca2+ signaling. Science $2010 ; 327: 1250-4$.

8. Shigetomi $\varepsilon$, Jackson-Weaver 0 , Huckstepp RT, et al. TRPAl channels are regulators of astrocyte basal calcium levels and long-term potentiation via constitutive D-serine release. J Neurosci $2013 ; 33: 10143-53$.

9. Takata N, Mishima T, Hisatsune C, et al. Astrocyte calcium signaling transforms cholinergic modulation to cortical plasticity in vivo. J Neurosci $2011 ; 31: 18155-65$.

10. Panatier A, Theodosis DT, Mothet JP, et al. Glia-derived D-serine controls NMDA receptor activity and synaptic memory. Cell $2006 ; 125: 775-84$.

11. Kakegawa W, Miyoshi Y, Hamase K, et al. D-serine regulates cerebellar LTD and motor coordination through the delta2 glutamate receptor. Nat Neurosci $2011 ; 14: 603-11$.

12. Woo DH, Han KS, Shim JW, et al. TREK-1 and Bestl channels mediate fast and slow glutamate release in astrocytes upon GPCR activation. Cell $2012 ; 151: 25-40$.

13. Han J, Kesner $P$, Metna-Laurent $M$, et al. Acute cannabinoids impair working memory through astroglial CB1 receptor modulation of hippocampal LTD. Cell $2012 ; 148: 1039-50$.

14. Min R, Nevian T. Astrocyte signaling controls spike timing-dependent depression at neocortical synapses. Nat Neurosci $2012 ; 15: 746-53$

15. Navarrete M, Perea G, Fernandez de Sevilla D, et al. Astrocytes mediate in vivo cholinergicinduced synaptic plasticity. PLoS Biol $2012 ; 10$ : e1001259.

16. Gordon GR, Iremonger KJ, Kantevari S, et al. Astrocyte-mediated distributed plasticity at hypothalamic glutamate synapses. Neuron 2009 ; 64 : 391-403.

17. Pascual 0 , Casper KB, Kubera C, et al. Astrocytic purinergic signaling coordinates synaptic networks. Science $2005 ; 310: 113-6$.

18. Gordon GR, Baimoukhametova DV, Hewitt SA, et al. Norepinephrine triggers release of glial ATP to increase postsynaptic efficacy. Nat Neurosci 2005 ; 8 : 1078-86.

19. Fields RD, Burnstock G. Purinergic signalling in neuron-glia interactions. Nat Rev Neurosci 2006 ; $7: 423-36$.

20. Chen J, Tan Z, Zeng L, et al. Heterosynaptic long-term depression mediated by ATP released from astrocytes. Glia $2013 ; 61: 178-91$.

21. Bazargani N, Attwell D. Astrocyte calcium signaling: the third wave. Nat Neurosci 2016 ; 19 : $182-9$

22. Hamilton NB, Attwell D. Do astrocytes really exocytose neurotransmitters? Nat Rev Neurosci $2010 ; 11: 227-38$

23. Valtcheva S, Venance L. Astrocytes gate Hebbian synaptic plasticity in the striatum. Nat Commun $2016 ; 7: 13845$

24. Omrani A, Melone M, Bellesi M, et al. Up-regulation of GLT-1 severely impairs LTD at mossy fibre-CA3 synapses. J Physiol $2009 ; 587: 4575-88$.

25. Filosa A, Paixao S, Honsek SD, et al. Neuron-glia communication via EphA4/ephrin-A3 modulates LTP through glial glutamate transport. Nat Neurosci $2009 ; 12$ : 1285-92.

26. Park H, Poo MM. Neurotrophin regulation of neural circuit development and function. Nat Rev Neurosci $2013 ; 14: 7-23$.

27. Vignoli B, Battistini G, Melani R, et al. Peri-synaptic glia recycles brain-derived neurotrophic factor for LTP stabilization and memory retention. Neuron $2016 ; 92: 873-87$.
28. Parkhurst CN, Yang G, Ninan I, et al. Microglia promote learning-dependent synapse formation through brain-derived neurotrophic factor. Cell 2013 $155:$ : 1596-609.

29. Roumier A, Bechade C, Poncer JC, et al. Impaired synaptic function in the microglial KARAP/DAP12-deficient mouse. J Neurosci 2004 ; 24 : 11421-8.

30. Barres BA. The mystery and magic of glia: a perspective on their roles in health and disease. Neuron $2008 ; 60: 430-40$.

31. Bechade C, Cantaut-Belarif $Y$, Bessis A. Microglial control of neuronal activity. Front Cell Neurosci $2013 ; 7: 32$.

32. Stellwagen D, Malenka RC. Synaptic scaling mediated by glial TNF-alpha. Nature $2006 ; 440: 1054-9$

33. Wang $Q$, Rowan MJ, Anwyl R. Beta-amyloid-mediated inhibition of NMDA receptor-dependent long-term potentiation induction involves activation of microglia and stimulation of inducible nitric oxide synthase and superoxide. J Neurosci $2004 ; 24: 6049-56$.

34. Zhang J, Malik A, Choi HB, et al. Microglial CR3 activation triggers long-term synaptic depression in the hippocampus via NADPH oxidase. Neuron 2014 ; 82 : 195-207.

35. Bechade C, Colasse S, Diana MA, et al. NOS2 expression is restricted to neurons in the healthy brain but is triggered in microglia upon inflammation. Glia $2014 ; 62$ : 956-63.

36. Biber K, Neumann H, Inoue K, Boddeke HW. Neuronal On and Off signals control microglia. Trends Neurosci 2007 ; 30 : 596-602.

37. Paolicelli RC, Bolasco G, Pagani F, et al. Synaptic pruning by microglia is necessary for normal brain development. Science $2011 ; 333: 1456-8$.

38. Hoek RM, Ruuls SR, Murphy CA, et al. Down-regulation of the macrophage lineage through interaction with OX2 (CD200). Science $2000 ; 290: 1768-71$

39. Costello DA, Lyons A, Denieffe $S$, et al. Long term potentiation is impaired in membrane glycoprotein CD200-deficient mice: a role for Toll-like receptor activation. J Biol Chem $2011 ; 286$ : 34722-32.

40. Nishiyama H, Knopfel T, Endo S, Itohara S. Glial protein S100B modulates long-term neuronal synaptic plasticity. Proc Natl Acad Sci USA 2002 ; 99 : 4037-42.

41. Gong $Q J$, Li YY, Xin WJ, et al. ATP induces long-term potentiation of C-fiberevoked field potentials in spinal dorsal horn: the roles of $\mathrm{P} 2 \mathrm{X} 4$ receptors and p38 MAPK in microglia. Glia 2009 ; 57 : 583-91.

42. Pascual $O$, Ben Achour $S$, Rostaing $P$, et al. Microglia activation triggers astrocyte-mediated modulation of excitatory neurotransmission. Proc Natl Acad Sci USA $2012 ; 109$ : ع197-205.

43. Domercq M, Brambilla L, Pilati $\varepsilon$, et al. P2Y1 receptor-evoked glutamate exocytosis from astrocytes: control by tumor necrosis factor-alpha and prostaglandins. J Biol Chem 2006 ; 281 : 30684-96.

44. Catalano M, Lauro C, Cipriani R, et al. CX3CLl protects neurons against excitotoxicity enhancing GLT-1 activity on astrocytes. J Neuroimmunol $2013 ; 263: 75-82$
TIRÉS À PART

R. Ronzano

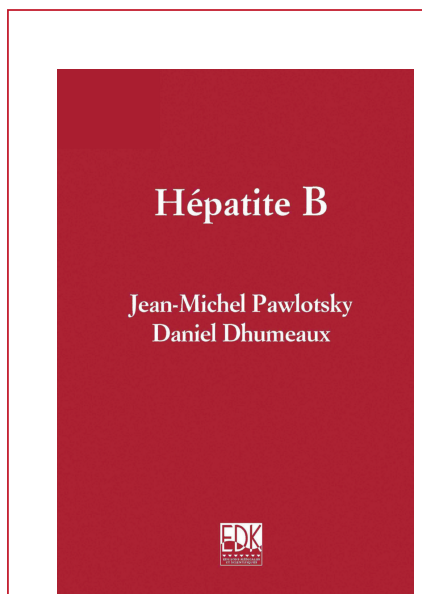

ISBN : 978-2-8425-4131-6 576 pages

\section{Bon de commande}

À retourner à EDP Sciences, 109, avenue Aristide Briand, 92541 Montrouge Cedex Tél. : 0141177405 - Fax : 0149850345 - E-mail : francois.flori@edpsciences.org NOM :

Adresse :

Code postal : Prénom :

Pays :

Fonction :

Je souhaite recevoir l'ouvrage Hépatite B : $54 €+3 €$ de port $=\mathbf{5 7} €$ TTC offre exceptionnelle réservée aux abonnés à $\mathrm{m} / \mathrm{s}$ jusqu'au 31 décembre 2010

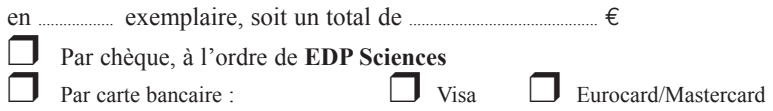

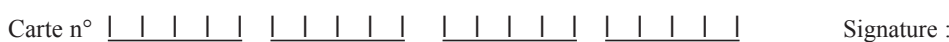

Date d'expiration :

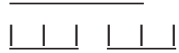

$\mathrm{N}^{\circ}$ de contrôle au dos de la carte : 